\title{
Direct Fabrication of Submicron-size Periodic Structures by Backside Irradiation
}

\author{
Tomoka Nakazumi, and Tadatake Sato

\begin{abstract}
Research Insitute for Sustainable Chemistry, National Institute of Advanced Industrial Science and Technology (AIST), Tsukuba Central 5, 1-1-1 Higashi, Tsukuba, Ibaraki 305-8565, Japan
\end{abstract} \\ E-mail: sato-tadatake@aist.go.jp
}

\begin{abstract}
Toward adding specific functions to materials surface, submicron-size periodic structures were fabricated onto glass substrates by photochemical polymerization. A laser diode with continuous wave at $405 \mathrm{~nm}$ was used as a light source for the polymerization. Backside laser irradiation was utilized for inducing the photochemical polymerization in the close vicinity of a liquid-solid interface. Reactive liquid mixture composed of pentaerythritol triacrylate and 2-isopropylthioxanthone, those are used as an acrylic monomer and a photoinitiator, respectively, was set onto an aminosilane coated glass substrate. To confine the region where the polymerization occurs in the vicinity of the liquid-solid interface, reactive mixture containing high concentration of photoinitiator was employed. Optical penetration depth was decreased to ca. $30 \mu \mathrm{m}$. This confinement is indispensable for the present method to fabricated submicron-size periodic structures. A laser beam was once divided into four beams. Then, two or four beams were used for backside irradiation with interference. Incident angles of each divided beam were set to be 12 degrees in all experiments. Upon two beam irradiation, fringe structures with a period of $0.9 \mu \mathrm{m}$ could be fabricated. Meanwhile, upon four beam irradiation, two-dimensional array of dot structures with a diameter of $0.7 \mu \mathrm{m}$ was successfully fabricated.

DOI: 10.2961/jlmn.2016.03.0006
\end{abstract}

Keywords: laser direct write, photochemical polymerization, diode laser, interference, backside irradiation, periodic surface microstructures

\section{Introduction}

Two-dimensional periodic structures with submicronsize have attracted much interest because such surface structures can provide specific functions, such as hydrophobicity, hydrophobicity-based self-cleaning, antireflection, and so on [1]. Interference of laser beams has often been used for fabricating such periodic structures [2-4].

A $405 \mathrm{~nm}$ diode laser can be applied for laser interference lithography as an economical light source [5, 6]. In these studies, performance of the laser was demonstrated to fabricate micro-periodic structures on a photoresist. However, patterns generated on a photoresist have to be applied to following etching process for generating the structures in real use.

A photocurable resin has been often used for fabricating three-dimensional structures in additive manufacturing. Photochemical polymerization is a base reaction of such fabrications [7-10]. Three-dimensional structures were fabricated directly within a photocurable resin by multi-photon lithography [7], while the fabrications on single-photon absorption were executed in a layer-by-layer approach because of the limitation arising from optical penetration depth [8]. Since photocurable resins are originally in liquid form, it is difficult to prepare the films with controlled thickness as in the case of photoresists.

For generating submicron-size periodic structures onto solid surface, localization of photo-induced process in the close vicinity of the surface is necessary. Backside irradiation through transparent substrates is one promising meth- od for such localization although the applicable substrates are limited to transparent ones. Due to optical penetration depth, the region where the photo-induced process occurs can be localized in the close vicinity of the surface of transparent substrate which is in contact with lightabsorbing media. Micromachining of transparent materials using the backside irradiation, called as laser-induced backside wet etching (LIBWE), has been developed as far [11] Among the studies on micromachining by this method, interference of laser beam was successfully used for generating periodic structures in micrometer-size [12, 13] or in sub-micrometer-size [14, 15].

In this work, photochemical polymerization at a liquidsolid interface was applied to fabricate submicron-size periodic structures on the solid surface. Backside laser irradiation is utilized for localization of photochemical polymerization at the interface.

\section{Experimental}

A laser diode with continuous wave at $405 \mathrm{~nm}$ (Crystal Laser, DL405-015-S) was employed as a light source for photochemical polymerization. The laser was operated at $15 \mathrm{~mW}$. The laser beam was expanded and spatiallyfiltered, and then split in four beams by means of three beam splitters as shown in Fig. 1(a). Irradiation power was adjusted by using a half-wave plate and a polarized beamsplitter. 
Each beam was set to be incident on the reactive liquid mixture through the glass substrate with a fixed incident angle $(\theta)$. Incident beams were set so that the irradiated areas overlap with each other at the interface as illustrated in Fig. 1(b). In this work, the incident angle was set to 12 degrees for all experiments.

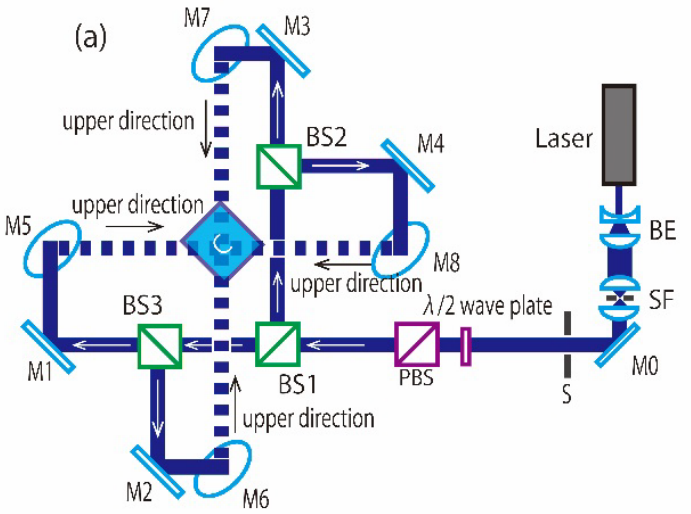

(b)

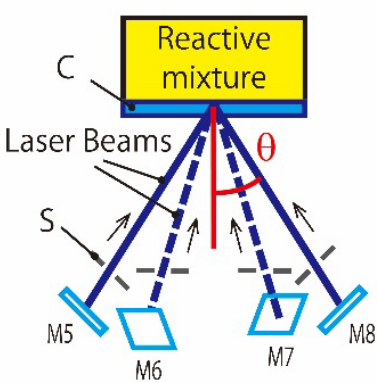

Fig. 1 (a) Top view of experimental setup for photochemical polymerization with interfering four beam in back-side irradiation layout and (b) configuration of backside irradiation in upward direction with interfering four beams. L: Laser, BE: Beam expander, SF: Spatial filter, W: $\lambda / 2$-plate, M: Mirror, $S$ : Shutter, PBS: Polarizing beamsplitter, BS: Beamsplitter, C: Substrate

Shutters set on mirros M5 M8 were employed for selection of beams for irradiation. Another shutter set before $\lambda / 2$-plate was used for control the irradiation time with a resolution of $0.1 \mathrm{~s}$.

Pentaerythritol triacrylate (Alfa Aeser) and 2isopropylthioxanthone (ITX) (Wako Chemical) were used as an acrylic monomer and a photoinitiator, respectively. This combination was employed in previous studies $[9,10]$. All chemicals were used without further purification. (a)<smiles>C=CC(=O)OCC(CO)(CO)COC(=O)CC</smiles>

(b)

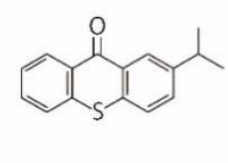

Fig. 2 Chemical structures of (a) pentaerythritol triacrylate and (b) 2-isopropylthioxanthone (ITX)

ITX was dissolved into the liquid acrylic monomer at concentrations of $0.16,1.5$, and $3.5 \mathrm{wt} \%$ and stirred for 2 hours for complete dissolution. Thus prepared reactive mixture was filtered by a syringe filter with a pore size of $0.45 \mu \mathrm{m}$ before irradiation.

Filtered reactive mixture was set onto an amino silane coated glass slide as depicted in Fig. 1(b). After irradiation for up to $1.0 \mathrm{~s}$, unreacted liquid mixture was removed from the glass slide, and then the glass slide was rinsed two times with ethanol. Fabricated structures were observed by means of a laser confocal microscope (Keyence, VK-8500) and a scanning electron microscope (SEM) (Keyence, VE7800).

\section{Results and Discussions}

\subsection{Photochemical process}

We employed a diode laser $(\lambda=405 \mathrm{~nm})$ as a light source for photochemical polymerization. Fig. 3 shows absorption spectrum of a reactive mixture containing 0.16 wt\% of ITX. The observed absorption band peaked at 386 $\mathrm{nm}$ was assigned to ITX.

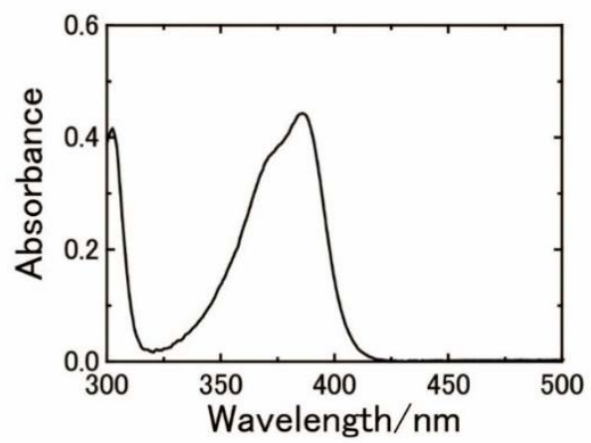

Fig. 3 Absorption spectrum of ITX dissolved in the acrylic monomer (0.16 wt \%) set within a $0.12 \mathrm{~mm}$ gap between two silica glass slides.

For the measurements, reactive mixtures were set within a gap between two silica slide glasses with the spacer of a thickness of $0.12 \mathrm{~mm}$. When a concentration of ITX was $0.16 \mathrm{wt} \%$, absorbance at irradiation wavelength of $405 \mathrm{~nm}$ was to be 0.022 . This absorbance correspond to the optical penetration depth of $2400 \mu \mathrm{m}$. For higher concentrations of ITX, corresponding penetration depths become smaller, meaning the region where photochemical reactions induced was confined within close vicinity of the liquid-solid interface. Estimated optical penetration depths in three cases were listed in Table 1.

Table 1 Absorbance and optical penetration depth of reactive mixtures

\begin{tabular}{ccc}
\hline $\begin{array}{c}\text { Concentration } \\
\text { of ITX }\end{array}$ & $\begin{array}{c}\text { Absorbance at } \\
\lambda=405 \mathrm{~nm}\end{array}$ & $\begin{array}{c}\text { Optical penetra- } \\
\text { tion depth }\end{array}$ \\
\hline 0.16 & 0.022 & $2400 \mu \mathrm{m}$ \\
1.6 & 0.645 & $81 \mu \mathrm{m}$ \\
3.5 & 1.569 & $33 \mu \mathrm{m}$ \\
\hline
\end{tabular}




\subsection{Two interfering beam irradiation}

First, two interfering beam irradiation generating interference fringes with a period of $\Lambda$ was examined, where $\Lambda$ can be calculated by equation (1).

$$
\Lambda=\frac{\lambda}{2 \sin \theta}
$$

Based on the incident angle setting to be 12 degrees, $\Lambda$ was calculated to be $0.97 \mu \mathrm{m}$. Power for each divided beam was set to $0.23 \mathrm{~mW}$. Thus, total power for two beam irradiation was to be $0.46 \mathrm{~mW}$ at the solid-liquid interface.

For the reactive mixture containing ITX with a concentration of $0.16 \mathrm{wt} \%$, generation of polymerized product on the glass surface was confirmed with irradiation for $17.8 \mathrm{~s}$ or longer. Thickness of the generated polymer was 3-4 $\mu \mathrm{m}$ at the center of the generated structure, where the smooth and homogeneous polymer surface was generated (right side in Figs. 4(a) and 4(b)). The structures reflecting interference fringes were only observed near edge region.

On the other hand, generation of fringe structures could be observed in the polymerized product generated from the reactive mixture containing $1.5 \mathrm{wt} \%$ of ITX. In this case, generation of the polymerized products onto substrates could be observed for irradiation time longer than $2.3 \mathrm{~s}$. Fringe structures with a period of $0.9 \mu \mathrm{m}$ could be partially observed. While many wider lines that would be made up of fused multiple lines were observed at the center of the product. When irradiation time was increased by $0.1 \mathrm{~s}$, generation of an unstructured polymer film was observed at the center of the product as in the case of $0.16 \mathrm{wt} \%$. Thus, fine structures disappeared by excess of dose.

Successful generation of fringe structures could be observed under irradiation for the reaction mixture containing $3.5 \mathrm{wt} \%$ of ITX. As shown in Fig. 4(e), periodic structures with a period of $0.9 \mu \mathrm{m}$ were observed within whole generated product. The irradiation time was reduced to $0.9 \mathrm{~s}$. For slight excess of dose, partial fusion of line structures started to be observed. Fig. 5 shows surface profile of the structures obtained by means of laser confocal microscope and SEM. The height of fringe structures was $0.2-0.3 \mu \mathrm{m}$ in these results. This height is much smaller than the optical penetration depth in Table 1.

Photochemical polymerization is initiated by photoexcitation of the photoinitiator, ITX (Fig. 6(a)); the excited ITX abstracts $\mathrm{H}$ from acrylic monomer to generate radical promoting polymerization. This initial reaction occurs within the region the light for excitation can reach. When the polymerization fully promotes in this region, the thickness of the polymerized parts could be related to the optical penetration depth [8]. In the present case, polymerization were not fully promoted for fabricating fine surface structures. The polymers generated within reactive mixture would form aggregation and deposit onto the substrate. When irradiation from front side for a thin liquid layer (layer thickness: ca. $110 \mu \mathrm{m}$ ) set on the substrate was examined, we could not find the conditions for obtaining the polymerized products on the substrate.

When optical penetration depth becomes smaller, the radicals would be generated at the positions more close to substrate surface with higher density. Increased density of radical generation would make the aggregation of polymer- ized products easier. Moreover, the polymerization near the solid surface promotes the deposition of the polymerized product onto surface.

Thus the high concentration of the photoinitiator contributes to the successful fabrication of surface microstructures by photochemical polymerization under backside irradiation.

(a)

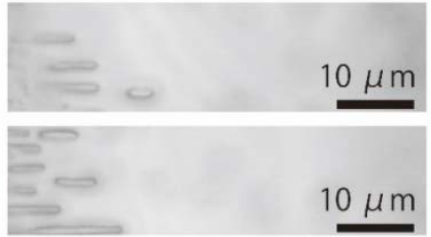

(c)

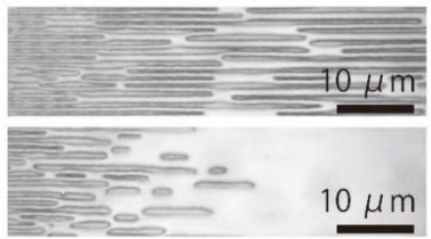

(e)

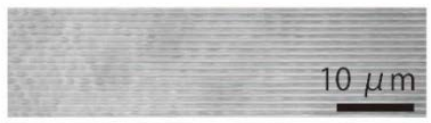

(f)

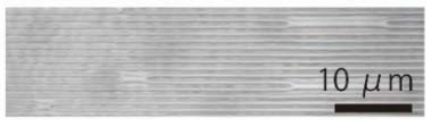

(g)

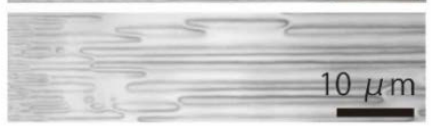

Fig. 4 Confocal laser micrographs of polymer microstructures fabricated on the glass plates by photochemical polymerization. Concentration of ITX and irradiation time were as follows: (a) $0.16 \mathrm{wt} \%$ and

$17.8 \mathrm{~s}$, (b) $0.16 \mathrm{wt} \%$ and $17.9 \mathrm{~s}$, (c) $1.5 \mathrm{wt} \%$ and 2.3

s, (d) $1.5 \mathrm{wt} \%$ and $2.4 \mathrm{~s}$, (e) $3.5 \mathrm{wt} \%$ and $0.9 \mathrm{~s}$, (f)

$3.5 \mathrm{wt} \%$ and $1.0 \mathrm{~s}$, (g) $3.5 \mathrm{wt} \%$ and $1.1 \mathrm{~s}$. (a)
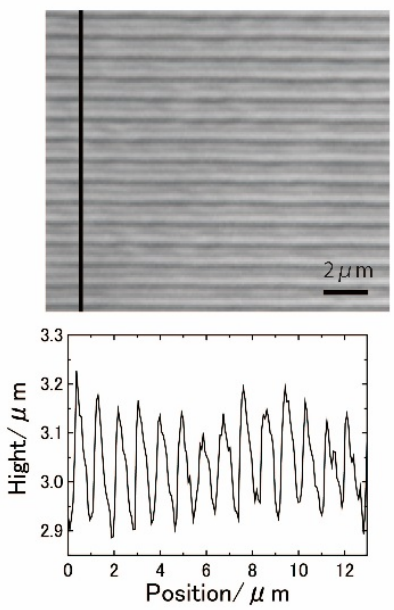

(b)

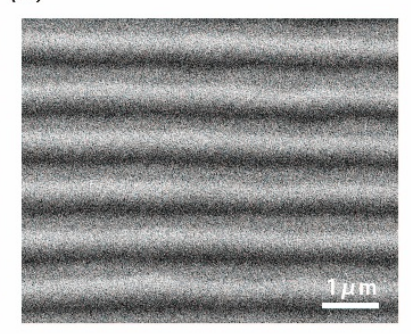

Fig. 5 (a) Confocal laser micrograph and surface profile of fringe structure generated from the reaction mixture with ITX concentration of 3.5 wt\% upon irradiation for 0.9 s. (b) A SEM image of the same structure 
(a)
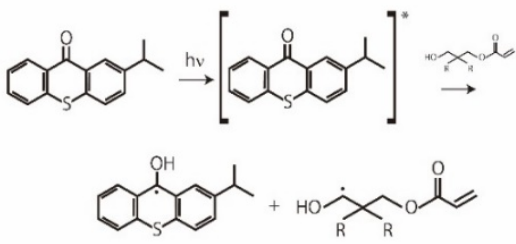

(b)
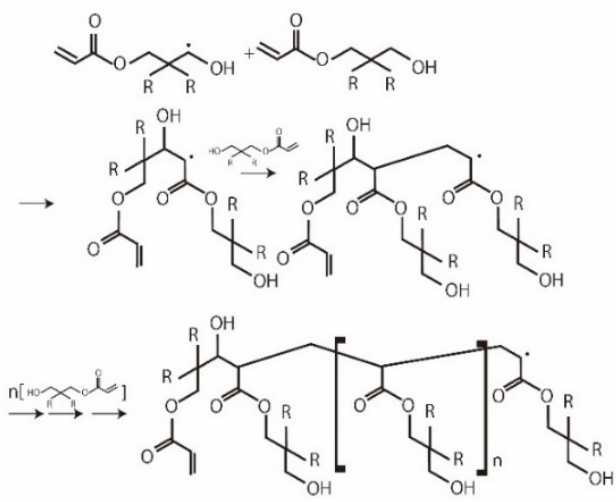

Fig. 6 Reaction scheme of (a) radical generation and (b) polymerization

\subsection{Four interfering beam irradiation}

Next, for fabricating two dimensional dot array structure, four interfering beam irradiation was examined by employing the reaction mixture containing $3.5 \mathrm{wt} \%$. In this case, power for each divided beam was set to $0.0325 \mathrm{~mW}$. Thus, total power for four beam irradiation was to be 0.13 $\mathrm{mW}$ at the solid-liquid interface.

In this case, the region for the photochemical polymerization was further confined two-dimensionally. The power of irradiation was reduced to control the fabrication. As a results, irradiation for $5.4 \mathrm{~s}$ resulted in successful fabrication of two-dimensional dot array structure as shown in Fig. 7. The diameter and the height of dot structures were around $0.7 \mu \mathrm{m}$ and $0.1 \mu \mathrm{m}$, respectively. Thus, confinement of the region where the photochemical polymerization occurs was effective to fabricate microstructures upon backside irradiation.

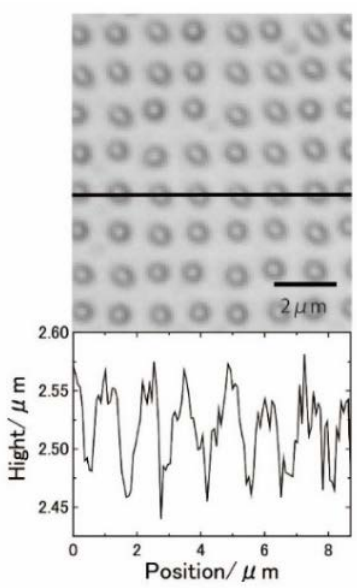

Fig. 7 Confocal laser micrograph and surface profile of two dimensional dot array fabricated on the basis of interference of four beams.

\section{Conclusion}

Periodic structures were fabricated by the photochemical polymerization induced upon backside irradiation. A laser diode with continuous wave at $405 \mathrm{~nm}$ was used for the backside laser irradiation for inducing the photochemical polymerization at a liquid-solid interface. To fabricate submicron-size periodic structures upon backside irradiation, confinement of the region where the photochemical reaction occurs is quite important. In this work, by employing the reactive mixture containing the photoinitiator ITX with a concentration of $3.5 \mathrm{wt} \%$, optical penetration depth was decreased to ca. $30 \mu \mathrm{m}$, resulting in the successful fabrication of periodic structures. Upon two beam irradiation, fringe structures with a period of $0.9 \mu \mathrm{m}$ could be fabricated. Meanwhile, upon four beam irradiation, twodimensional array of dot structures with a diameter of 0.7 $\mu \mathrm{m}$ was successfully fabricated.

\section{Acknowledgments}

This work was supported in part by Grants-in-Aid for Young Scientists (B) (No. 16K17493) from the Ministry of Education, Culture, Sports, Science and Technology, Japan.

\section{References}

[1] K. -C. Park, H. J. Choi, C.-H. Chang, R. E. Cohene, G. H. Mckinley and G. Barbastathis: ACS Nano, 6, (2012) 3789.

[2] H. H. Solak: J. Phys. D: Appl. Phys., 39, (2006) R171.

[3] Y. Nakata, N. Miyanaga and T. Okada: Appl. Surf. Sci., 253, (2007) 6555.

[4] D. Nakamura, T. Simogaki, K. Okazaki, M. Higashihata and T. Okada: J. Laser Micro/Nanoeng., 8, (2013) 206.

[5] C. P. Fucetola, H. Korre and K. K. Berggren: J. Vac. Sci. Technol. B, 27, (2009) 2958.

[6] I. Byun and J. Kim: J. Micromech. Microeng., 20, (2010) 055024.

[7] G. Von Freymann, A. Ledermann, M. Thiel, I. Staude, S. Essing, K. Busch and M. Wegener: Adv. Funct. Mater., 20, (2010) 1038.

[8] A. Bertsch, J. Y. Jézéquel, and J. C. André: J. Photochem. Photobiol. A: Chem., 107, (1997) 275.

[9] B. Harke, P. Bianchini, F. Brandi and A Diaspro: Chem. Phys. Chem., 13, (2012) 1429.

[10]B. Harke, W. Dallari, G. Grancini, D. Fazzi, F. Brandi A. Petrozza and A Diaspro: Adv. Mater., 25, (2012) 904.

[11]T. Sato, Y. Kawaguchi, R. Kurosaki, A. Narazaki, W. Watanabe and H. Niino: J. Laser Micro/Nanoeng., 6, (2011) 204, and references therein.

[12] K. Zimmer, R. Bohme, A.Braun, B. Rauschenbach and F. Bigl: Appl. Phys. A, 74, (2002) 453.

[13] T. Nakazumi, T. Sato, A. Narazaki and H. Niino: J. Micromech. Microelectro., submitted.

[14]C. Vass, K. Osvay and B. Hopp: Opt. Express, 14, (2006) 8354.

[15] C. Vass, K. Osvay, B. Hopp and Z. Bor: Appl. Phys. A, 87, (2007) 611.

(Received: May 20, 2016, Accepted: August 22, 2016) 